Physics

Physics Research Publications

Purdue University

Year 2005

\author{
Radio-X-ray correlation and the \\ "quiescent state" of black hole sources \\ F. Yuan W. Cui
}

This paper is posted at Purdue e-Pubs.

http://docs.lib.purdue.edu/physics_articles/253 
The Astrophysical Journal, 629:408-413, 2005 August 10

(C) 2005. The American Astronomical Society. All rights reserved. Printed in U.S.A.

\title{
RADIO-X-RAY CORRELATION AND THE “QUIESCENT STATE” OF BLACK HOLE SOURCES
}

\author{
Feng Yuan and Wei Cui \\ Department of Physics, Purdue University, 525 Northwestern Avenue, West Lafayette, IN 47907; \\ fyuan@physics.purdue.edu, cui@physics.purdue.edu \\ Received 2004 November 30; accepted 2005 April 28
}

\begin{abstract}
Recently a correlation between the radio and X-ray luminosities, $L_{R} \propto L_{\mathrm{X}}^{0.7}$, was found in black hole sources including black hole candidates in our Galaxy and active galactic nuclei. We show that the correlation can be understood in the context of an accretion-jet model developed for explaining the spectral and timing properties of XTE J1118+480. More importantly, we show that when the X-ray luminosity is below a critical value, $\lesssim\left(10^{-5}\right.$ to $\left.10^{-6}\right) L_{\mathrm{Edd}}$, if the jet persists, the correlation should turn and become steeper, $L_{R} \propto L_{\mathrm{X}}^{1.23}$, and the X-radiation of the system should be dominated by the emission from the jet, rather than by the accretion flow. Possible observational evidence for our predictions is presented, and future observations to further test our predictions are proposed.
\end{abstract}

Subject headings: accretion, accretion disks — black hole physics — galaxies: active — ISM: jets and outflows — X-rays: stars

\section{INTRODUCTION}

In the low/hard state, the radio spectrum of black hole candidates (BHCs) is usually flat or even inverted, which is often taken as evidence for the presence of jets (Fender 2005 and references therein). This is because, on the one hand, such a spectrum is characteristic of jets in active galactic nuclei (AGNs; e.g., Blandford \& Königl 1979), and on the other hand, it is difficult to explain it by invoking emission from the underlying accretion flow (Yuan et al. 2005, hereafter YCN05). A strong correlation between the radio and X-ray luminosities of BHCs in the hard state has been found recently (Corbel et al. 2003; Gallo et al. 2003): $L_{R} \propto L_{\mathrm{X}}^{0.7}$, where $L_{R}$ is the radio luminosity at $8.6 \mathrm{GHz}$ and $L_{\mathrm{X}}$ is the $2-11 \mathrm{keV}$ $\mathrm{X}$-ray luminosity. The correlation extends more than three decades in $L_{\mathrm{X}}$, from $L_{\mathrm{X}} \gtrsim 10^{-5} L_{\mathrm{Edd}}$ to $L_{\mathrm{X}} \lesssim 10^{-2} L_{\text {Edd }}\left(L_{\mathrm{Edd}}\right.$ is the Eddington luminosity). The lowest luminosity is close to the quiescent state luminosity of V404 Cyg but is still much higher than that of typical BHCs (e.g., Kong et al. 2002; McClintock et al. 2003). The correlation also holds for AGNs (Merloni et al. 2003, hereafter MHD03; Falcke et al. 2004).

It is generally thought that the X-ray emission from $\mathrm{BHCs}$ originates in the accretion flows (see a review by Narayan 2005). The observed radio-X-ray correlation strongly implies a casual connection between the accretion flow and jets. Recently, coupled accretion-jet models have been proposed and applied to the hard state of XTE J1118+480, a source for which we have almost the best spectral and timing observational results among all BHCs (YCN05; Malzac et al. 2004). In these models, the accretion flow is composed of an inner advectiondominated accretion flow (ADAF)-like hot accretion flow and an outer standard thin disk (Esin et al. 1997; Malzac et al. [2004] also discuss the possibility that the X-rays may come from a patchy corona rather than an ADAF). An additional jet component is assumed to form at the innermost region of the accretion flow. The X-ray emission is dominated by the hot accretion flow, while the radio emission comes from the jet. The accretion-jet models not only can explain the broadband spectral energy distribution of XTE J1118+480 ranging from radio to X-ray (YCN05) but also can account for most of its complicated timing features (YCN05; Malzac et al. 2004).

It is natural to ask whether the models can explain the observed radio-X-ray correlation in a quantitative manner or what constraints the correlation can put on the models. One critical parameter in the problem is how the fraction of the accreted matter that goes into the jet, $\eta\left(\equiv \dot{M}_{\text {jet }} / \dot{M}\right)$, changes with $\dot{M}$. Unfortunately, no good theoretical constraint can be put on this fraction due to our poor understanding of the jet formation. Assuming $\eta$ is constant, MHD03 find that an ADAF jet model is roughly consistent with the observations at the $3 \sigma$ level.

In this paper we begin by investigating this question again. Instead of assuming a constant $\eta$, we investigate what functional form of $\eta(\dot{M})$ is required to explain the radio-X-ray correlation (§2.1). Such a study may supply us with some clue as to the jet formation mechanism. In $\S \S 2.2$ and 2.3, we investigate what the correlation will be below the lowest observed X-ray luminosity $\left(\sim 10^{-5} L_{\text {Edd }}\right)$. We find that when $L_{\mathrm{X}} \lesssim 10^{-5}$ to $10^{-6} L_{\text {Edd }}$, the radio-X-ray correlation will become much steeper, $L_{R} \propto L_{\mathrm{X}}^{1.23}$, and the X-ray emission of the source should be dominated by the jet, rather than by the accretion flow.

\section{MODEL}

\subsection{Radio-X-Ray Correlation in the Context of Accretion-Jet Model}

There are some uncertainties in the normalization of the observed radio-X-ray correlation (e.g., Gallo et al. 2003). Without losing generality, in our calculation we determine the normalization from the observed outburst-state radio and X-ray fluxes of XTE J1118+480 (see YCN05 for the observational data). Then the observed correlation is expressed as

$$
\left(\frac{L_{R}}{L_{\mathrm{Edd}}}\right)=10^{-7.34}\left(\frac{L_{\mathrm{X}}}{L_{\mathrm{Edd}}}\right)^{0.7} .
$$

This is shown by the segment $\mathrm{AB}$ in Figure 1. The point $\mathrm{A}$ corresponds to the outburst state of XTE J1118+480, and the point $\mathrm{B}$ is the lowest X-ray luminosity to which the observed correlation in BHCs extends, $\sim 10^{-5.2} L_{\text {Edd }}$ (Gallo et al. 2003).

The details of the accretion-jet model are described in YCN05. Briefly, the accretion flow is described as a geometrically thin cool disk outside a transition radius and a geometrically thick hot accretion flow (i.e., ADAF) inside the transition radius. The effect of outflow/convection is taken into account in calculating 


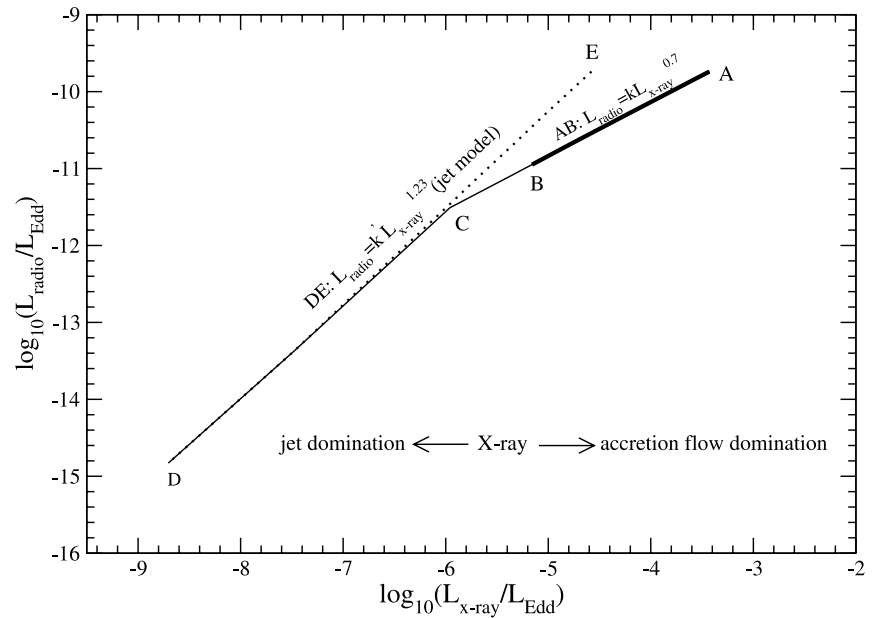

FIG. 1.-Radio $(8.6 \mathrm{GHz})-\mathrm{X}$-ray $(2-11 \mathrm{keV})$ correlation for BHCs. The observed correlation is shown by the segment AB. Segments BCD show the predicted correlation at lower luminosities, which approaches that of a pure-jet model, as shown by the segment DE. Note that below the point $\mathrm{C}\left(\sim 10^{-6} L_{\mathrm{Edd}}\right)$, the $\mathrm{X}$-ray emission is dominated by the jet and the correlation steepens.

the dynamics of the hot accretion flow. The main parameters are $\alpha=0.3, \beta=0.9$, and $\delta=0.5$. Near the black hole, we assume that a fraction of the accretion flow, $\eta$, is transferred into the vertical direction to form a jet. The half-opening angle of the jet is $\phi=0.1$, and the bulk Lorentz factor is $\Gamma_{j}=1.2$. Within the jet, internal shocks occur due to the collision of shells with different velocities. These shocks accelerate a fraction of the electrons into a power-law energy distribution. The steady-state energy distribution of the accelerated electrons is self-consistently determined, taking into account the effect of radiative cooling. Following the widely adopted approach in the study of gammaray bursts (GRBs), the energy density of accelerated electrons and amplified magnetic field is determined by two parameters, $\epsilon_{e}$ and $\epsilon_{B}$, which describe the fraction of the shock energy going into electrons and the magnetic field, respectively. The values of $\epsilon_{e}$ and $\epsilon_{B}$ are 0.06 and 0.02 , respectively, which are well within the typical range obtained in GRB afterglows (see YCN05 for details). We then calculate the synchrotron emission from these accelerated electrons. Of course, like any other jet models published in the literature, our jet model is only phenomenological because the physics of jet formation is still poorly understood.

We calculate the values of $L_{R}$ and $L_{\mathrm{X}}$ at various $\dot{M}$, adjusting $\eta$ so that equation (1) can be satisfied. The radio luminosity $L_{R}$ is always dominated by the emission from the jet while $L_{\mathrm{X}}$ is the sum of the emissions from the accretion flow (by thermal Comptonization and bremsstrahlung emissions) and jet (by optically thin synchrotron emission). We assume that the intrinsic physics of the accretion and jet does not depend on $\dot{M}$, so all the other model parameters are fixed in the process. Since outflow and convection are taken into account in our model, $\dot{M}$ is a function of radii. We therefore define $\eta \equiv \dot{M}_{\text {jet }} / \dot{M}\left(5 R_{\mathrm{S}}\right)$, where $\dot{M}\left(5 R_{\mathrm{S}}\right)$ is the accretion rate at $5 \mathrm{Sch}$ warzschild radii. When the luminosity is relatively high, such as at the point A in Figure $1, L_{\mathrm{X}}$ is dominated by the accretion flow (ref. Fig. 2 in YCN05). With the decrease of $\dot{M}$, however, the contribution of the jet to $L_{\mathrm{X}}$ becomes more and more important. This is because $\mathrm{X}$-ray emission from the accretion flow scales as $L_{\mathrm{X} \text {, acc }} \propto \dot{M}^{q}$ with $q \sim 2$ (see below for details), while that from the jet is due to the optically thin synchrotron emission and thus $L_{\mathrm{X}}$,jet $\propto \dot{M}$ (e.g., Heinz 2005). The radio luminosity is always dominated by the jet.

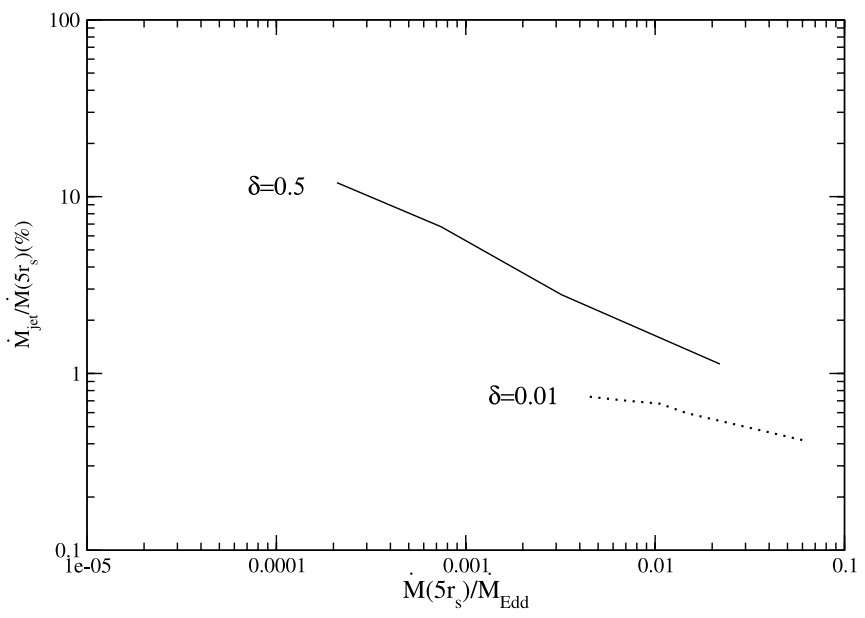

FIG. 2.-Ratio of the mass-loss rate in the jet $\left(\dot{M}_{\text {jet }}\right)$ to the accretion rate of the ADAF at $\sim 5 r_{\mathrm{S}}\left[\dot{M}\left(5 r_{\mathrm{S}}\right)\right]$ as a function of the accretion rate. The solid and dashed lines show results for two values of $\delta$ (the fraction of the viscously dissipated energy in directly heating electrons in an ADAF).

We find that our results are not sensitive to any model parameters except $\delta$, which describes the fraction of the viscously dissipated energy in directly heating electrons in the hot accretion flow (YCN05). We first consider the case of $\delta=0.5$, the value required in the detailed modeling of Sgr $\mathrm{A}^{*}$ by the most up-to-date ADAF model (Yuan et al. 2003). The solid line in Figure 2 shows the dependence of $\eta$ on $\dot{M}$. We can see from the figure that in this case to fit the radio-X-ray correlation, $\eta$ must be a strongly decreasing function of $\dot{M}$. If $\eta=$ constant, the predicted radio-X-ray correlation index would be $\xi_{\mathrm{RX}} \sim 1.3-1.4 \gg$ 0.7. This seems to be at odds with the result of MHD03. We find that the discrepancy is mainly due to their adoption of a smaller $\delta(=0.3)$. Following the notation in MHD03, if $L_{R} \propto \dot{M}^{\xi_{M}}$ and $L_{\mathrm{X}, \text { acc }} \propto \dot{M}^{q}\left(L_{\mathrm{X}, \text { acc }}\right.$ is the X-ray luminosity emitted from the accretion flow), the correlation index $\xi_{\mathrm{RX}}=\xi_{\dot{M}} / q=1.4 / q\left(\xi_{\dot{M}}=\right.$ 1.4; see Heinz \& Sunyaev 2003), if $\eta$ is assumed to be constant. MHD03 find that $q \sim 2.3$ for $\delta=0.3$. We do the calculations using $\delta=0.3$ and find that our result is in general agreement with MHD03. But for $\delta=0.5$, we find $q \approx 1.1,1.4$, and 1.8 for $\dot{M}$ in the ranges $\left[5 \times 10^{-2}, 2.5 \times 10^{-3}\right],\left[2.5 \times 10^{-3}, 5 \times 10^{-4}\right]$, and $[5 \times$ $\left.10^{-4}, 1 \times 10^{-4}\right]$ in units of $\dot{M}_{\text {Edd }}$, respectively. The reason for the difference in $q$ for different $\delta$ is as follows. The value of $L_{\mathrm{X} \text {, acc }}$ depends on the density $n_{e}$ and temperature $T_{e}$. With the decreasing of $\dot{M}$, the density decreases but $T_{e}$ increases. For larger $\delta, T_{e}$ increases faster; thus, $q$ is smaller. For comparison, we also calculate the case of $\delta=0.01$ and find that $\eta$ is nearly constant. In this case, $q \sim 2.4$, so the correlation index $\xi_{\mathrm{RX}}=1.4 / q \sim 0.6$ for a constant $\eta$, which is very close to 0.7 . Another reason for the discrepancy between our result and that of MHD03 is that we take into account the contributions of both the accretion flow and jet (MHD03 attribute $L_{X}$ only to the accretion flow), which results in a smaller "effective" $q$. The third (minor) reason is that we consider the effects of outflow and convection.

\subsection{The Steepening of Correlation and the Quiescent State of BHCs}

In the following we investigate the correlation below the point $\mathrm{B}$ in Figure 1. We assume that the jet persists and that the physics of jets does not change significantly at low luminosities. We extrapolate the derived $\eta(\dot{M})$ (which is approximately a power law) to lower $\dot{M}$ and calculate $L_{R}$ and $L_{\mathrm{X}}$ for different $\dot{M}$. The segments BCD in Figure 1 show our predicted 

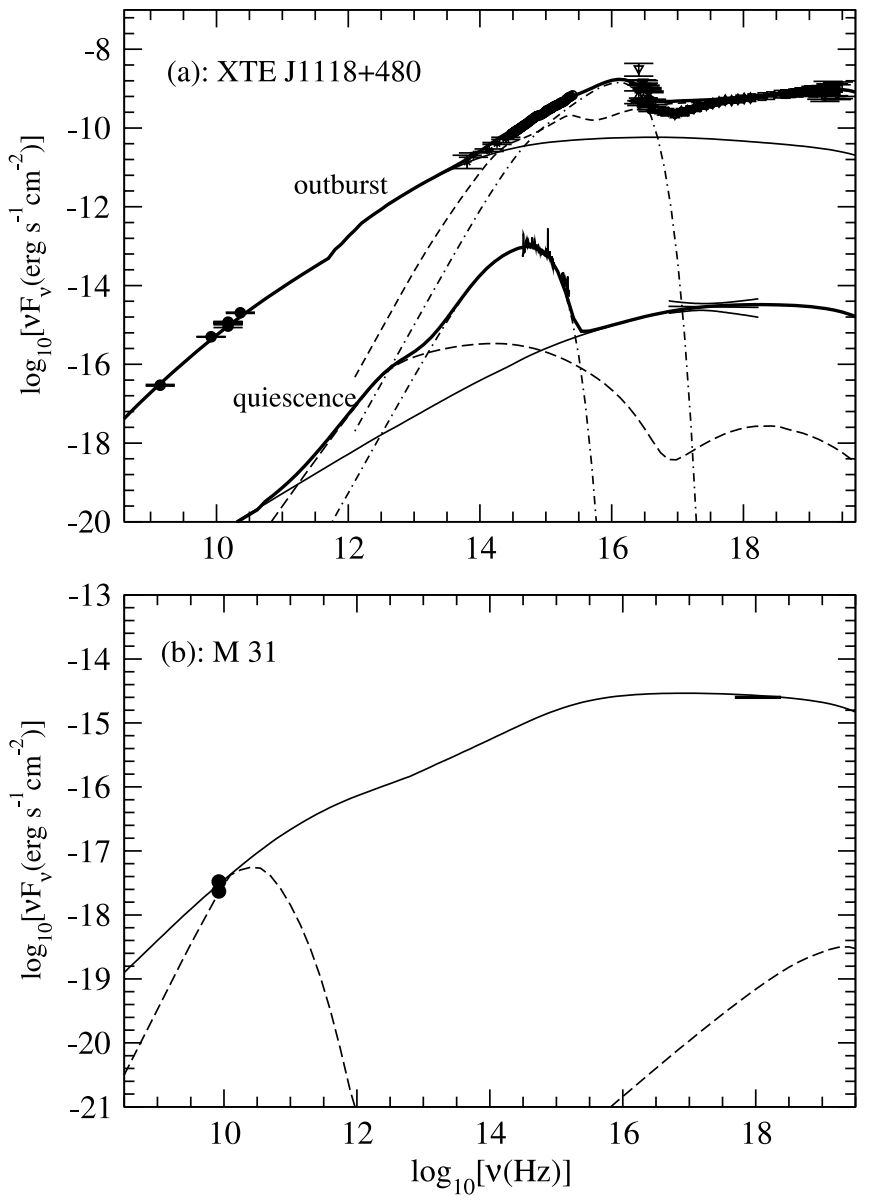

FIG. 3.-Accretion-jet model for two "quiescent" black hole sources. (a) Quiescent state of XTE J1118+480. The (optical and X-ray) data are from McClintock et al. (2003). The thin solid line shows the emission of the jet, the dashed line shows that of the $\operatorname{ADAF}(\delta=0.5)$, and the dot-dashed line shows that of a multitemperature blackbody component (e.g., a truncated disk; see McClintock et al. 2003 for details). Their sum is shown by the thick solid line. The parameters are $\dot{M}_{\text {jet }}=6 \times 10^{-8} \dot{M}_{\text {Edd }}$ and $\eta=15 \%$. Note that the X-ray emission is dominated by the jet. The model for the outburst state (YCN05) is also presented for comparison purposes. (b) "Quiescent" AGN, or M31. The radio data are from Crane et al. (1992, 1993), and the X-ray flux is from Garcia et al. (2005). A power-law X-ray spectrum with photon index of 2 is assumed. The solid line shows the emission of the jet, while the dashed line shows that for the $\operatorname{ADAF}(\delta=0.01)$. The parameters are $\dot{M}_{\text {jet }}=5 \times 10^{-9} \dot{M}_{\text {Edd }}$ and $\eta=1 \%$. Again, the X-ray emission is dominated by the jet.

radio-X-ray correlation at low $\dot{M}$. It is interesting to see that below a certain luminosity, represented by the point $\mathrm{C}$ in Figure 1, the correlation deviates from the extrapolation of the observed radio-X-ray correlation and approaches asymptotically the segment DE,

$$
\left(\frac{L_{R}}{L_{\mathrm{Edd}}}\right)=10^{-4.1}\left(\frac{L_{\mathrm{X}}}{L_{\mathrm{Edd}}}\right)^{1.23}
$$

The segment DE shows the correlation of a pure jet model, with the radio/X-ray emission being due to the optically thick/ thin synchrotron emission of the electrons in the jet. The normalization of the segment DE is determined by the results of modeling the outburst state of XTE J1118+480 (YCN05). (The point $\mathrm{D}$ represents the emission from the jet in XTE J1118+480 at the quiescent state; see Fig. $3 a$.) The correlation changes because, as we stated above, the contribution of the jet to the total X-ray emission becomes more and more important compared to that of the accretion flow as $\dot{M}$ becomes smaller. Below a certain $\dot{M}$ (the point $\mathrm{C}$ in Fig. 1), the X-ray luminosity will be completely dominated by the jet, and thus the correlation of the system will follow that of the pure jet model. This prediction is particularly relevant to the quiescent state of BHCs, because their X-ray luminosity is typically $\leqslant 10^{-6} L_{\mathrm{Edd}}$ (Kong et al. 2002; McClintock et al. 2003). We discuss this further in $\S 3$.

The index of the correlation of a pure jet model is $\sim 1.23$. This is in general agreement with Heinz (2005), who obtained $\xi_{\mathrm{RX}, \text { jet }} \approx 1.4$. On the other hand, Markoff et al. (2003) obtained $\xi_{\mathrm{RX}, \text { jet }} \approx 0.7$. We find that the discrepancy is mainly because Markoff et al. did not take into account the cooling break in the electrons' energy distribution, as was also pointed out by Heinz (2005). ${ }^{1}$ We should note that our results are not very sensitive to the exact form of $\eta$. We examine the two cases, $\delta=0.5$ and 0.01 , as well as one in which the value of $\eta$ at the lowest $\dot{M}$ in Figure 2 is used. We find that the result remains qualitatively the same, although the exact location of the point $\mathrm{C}$ and the slope of the segment BC in Figure 1 are slightly different. So we conclude that the change in slope from $\mathrm{AB}$ to $\mathrm{DE}$ is robust. The location of the intersection point $\mathrm{C}$ mainly depends on the normalization of the two segments. For different BHCs, the normalization of $\mathrm{AB}$ may vary by a factor of $\sim 5$ (Gallo et al. 2003). The uncertainties in the normalization of DE have two origins. One is in the jet model for a single source. Unlike the accretion flow, the jet parameters are not well constrained, and there is some degeneracy. However, we find that because of the excellent observational data of XTE J1118+480, the resulting uncertainty in the normalization is not large. The uncertainty from various sources depends on the diversity in the jet properties, such as its velocity. These quantities are currently poorly constrained, but we feel they should not differ too much among various sources.

\subsection{Extension from BHCs to AGNS}

While the radio-X-ray correlation index does not depend sensitively on the black hole mass, $M,{ }^{2}$ as shown by Heinz (2005), the normalization does. To extend our result to AGNs, we need the dependence of the correlation on the mass of the black hole. At relatively high luminosities (above the point $\mathrm{C}$ in Fig. 1), MHD03 found from analyzing a sample of AGNs and BHCs,

$\log L_{R}=(0.6 \pm 0.11) \log L_{\mathrm{X}}+0.78_{-0.09}^{+0.11} \log \left(M / M_{\odot}\right)+7.33_{-4.07}^{+4.05}$.

Neglecting the uncertainties, we rewrite this correlation as

$$
\log \left(\frac{L_{R}}{L_{\mathrm{Edd}}}\right)=0.6 \log \left(\frac{L_{\mathrm{X}}}{L_{\mathrm{Edd}}}\right)+0.38 \log \left(\frac{M}{M_{\odot}}\right)-7.926 .
$$

Equation (4) is almost identical to equation (1) but with an additional dependence on $M$ included.

Using a jet model, Heinz (2005) investigated the correlation index between $L_{R}$ and $M, \xi_{\mathrm{RM}}$, jet, and found that $\xi_{\mathrm{RM}, \text { jet }} \sim 0$.

1 The effect of the cooling break is correctly included in other jet models, e.g., Markoff et al. (2001).

2 From BHCs to AGNs, the synchrotron peak from the ADAF will move from optical to radio. Depending on the value of $\eta$, the contribution of the ADAF to $L_{R}(8.6 \mathrm{GHz})$ could become important when $L_{\mathrm{X}}$ is very low. In this case, the correlation index will become smaller. At frequencies far below $8.6 \mathrm{GHz}$, however, this effect is not important. 
We use our pure jet model to calculate the value of $\xi_{\mathrm{RM}}$, jet. ${ }^{3}$ We calculate the radio luminosity $L_{R}$ at various $M$, adjusting $\dot{M}_{\text {jet }}$ to keep $L_{\mathrm{X}}$ constant, and obtain the value of $\xi_{\mathrm{RM} \text {, jet }}=$ $\partial \log L_{R} / \partial \log M \sim 0.25$. This result is similar to that obtained by Heinz. Therefore, equation (2) can be generalized as

$$
\log \left(\frac{L_{R}}{L_{\mathrm{Edd}}}\right)=1.23 \log \left(\frac{L_{\mathrm{X}}}{L_{\mathrm{Edd}}}\right)+0.488 \log \left(\frac{M}{M_{\odot}}\right)-4.53,
$$

or equivalently,

$$
\log L_{R}=1.23 \log L_{\mathrm{X}}+0.25 \log \left(M / M_{\odot}\right)-13.45 .
$$

This equation describes the segment DE in Figure 1, with the additional dependence on $M$ included. From equations (4) and (5), we can estimate the X-ray luminosity at the point $\mathrm{C}$ (which is very close to the intersection point in Fig. 1)

$$
\log \left(\frac{L_{\mathrm{X}, \text { crit }}}{L_{\mathrm{Edd}}}\right)=-5.356-0.17 \log \left(\frac{M}{M_{\odot}}\right) .
$$

\section{OBSERVATIONAL TESTS}

\subsection{Radio-X-Ray Correlation at Low Luminosities}

Jonker et al. (2004) obtained (nearly) simultaneous radio and X-ray fluxes of XTE J1908+094 during the decaying phase of an X-ray outburst. Their X-ray measurements were taken on 2003 March 23, April 19, and May 13, but the radio measurements were taken only on March 25 and April 12. We fit the $\mathrm{X}$-ray fluxes with a parabola and estimate the X-ray flux for April 12 from the best-fit curve. Similarly, we obtain the radio flux for March 23 by linearly interpolating the March 25 and April 12 measurements. From the measured and estimated radio and X-ray fluxes for March 23 and April 12, we derive a radio$\mathrm{X}$-ray correlation index, $\xi_{\mathrm{RX}} \approx 1.28$, which is significantly different from $\xi_{\mathrm{RX}} \approx 0.7$. $^{4}$ Jonker et al. (2004) speculated that the discrepancy may imply that, unlike other BHCs, the accretion flow in this source is in the form of a standard thin disk rather than an ADAF, even at low luminosities.

Given that our predicted value for the correlation index is consistent with the range allowed by the J1908+094 data, however, we believe that a more likely scenario for the steeper radio-X-ray correlation is that the X-ray emission of XTE $\mathrm{J} 1908+094$ is already dominated by the jet at the observed $\mathrm{X}$-ray fluxes. If our explanation is correct, the X-ray luminosity of the source would have to be below the critical value (as indicated by the point $\mathrm{C}$ in Fig. 1), i.e., $L_{\mathrm{X}} \lesssim L_{\mathrm{X} \text {. crit }} \approx 10^{-5.5} L_{\mathrm{Edd}}$. The mass of the compact object in XTE J1908+094 is not known. Assuming a mass of $10 M_{\odot}$, we found that its X-ray luminosity would be $L_{\mathrm{X}} \sim 8 \times 10^{-4}(d / 8.5 \mathrm{kpc})^{2} L_{\text {Edd }}$ on March 23 and $\sim 3 \times 10^{-4}(d / 8.5 \mathrm{kpc})^{2} L_{\mathrm{Edd}}$ on April 12, which implies that the source would have to be very nearby, $d \sim 1 \mathrm{kpc}$. It remains to

\footnotetext{
${ }^{3}$ Our jet model developed in YCN05 also works for large $M$, because the basic physics of the jet should not depend on $M$, and the dependence of quantities (such as the frequency of the cooling break) on $M$ have been selfconsistently taken into account in our jet code.

${ }^{4}$ If we estimate the X-ray flux on April 12 by linearly interpolating the March 25 and April 19 (or April 19 and May 13) measurements, the correlation index would be $\xi_{\mathrm{RX}} \approx 1.48$ (or 1.0). If we estimated the X-ray flux on April 12 by assuming an exponential decay in X-ray flux with time between March 23 and April 19, as Jonker et al. (2004) did, the correlation index would be $\xi_{\mathrm{RX}} \approx 1.12$
}

be seen whether this is the case. At present, the distance to the source is poorly constrained, as pointed out by Jonker et al. (2004). We should stress that due to the uncertainty in the location of the point $\mathrm{C}$, the uncertainty in $d$ is significant.

As for AGNs, the observations of M31 seem to provide evidence that supports our predictions. The source was not included in the sample used by MHD03, presumably because the X-ray data were not available at the time. In this source, the mass of the black hole is $10^{7.5} M_{\odot}$. The radio luminosity of the source (at $3.6 \mathrm{~cm}$ ) is $10^{32.2}$ and $10^{32.37} \mathrm{ergs} \mathrm{s}^{-1}$ based on two different observations (Crane et al. 1992, 1993). The X-ray luminosity is very weak, $L_{\mathrm{X}} \sim 10^{35.5} \mathrm{ergs} \mathrm{s}^{-1} \sim 10^{-3.5} L_{\mathrm{X} \text {, crit }}(\sim 2.5 \sigma$ detection; Garcia et al. 2005). So this source is very appropriate for testing our prediction. From $L_{\mathrm{X}}$ and $M$, equation (3) predicts that the radio luminosity is $\sim 10^{34.45} \mathrm{ergs} \mathrm{s}^{-1}$, which is $\sim 100$ times higher than the observed value, while equation (6) predicts a value of $\sim 10^{32.2} \mathrm{ergs} \mathrm{s}^{-1}$, which is in good agreement with the observation. The spectral fitting result is shown in Figure $3 b$. In addition, Garcia et al. (2005) estimated the value of $\dot{M}$ to be $\dot{M} \sim 6 \times 10^{-6} \dot{M}_{\text {Edd }}$. But the X-ray luminosity predicted by an ADAF with such a $\dot{M}$ is only $\sim 10^{31} \mathrm{ergs} \mathrm{s}^{-1}$, which is about 4 orders of magnitude lower than the observed value, as shown by the dashed line in Figure $3 b$. On the other hand, we find that to produce the observed $L_{\mathrm{X}}$ by a jet, the required $\dot{M}_{\text {jet }} \sim$ $5 \times 10^{-9} \dot{M}_{\text {Edd }} \ll 6 \times 10^{-6} \dot{M}_{\text {Edd }}$, which is reasonable. Of course, the X-ray detection by Garcia et al. (2005) needs confirmation.

Most sources in the sample of MHD03 are observed at relatively high X-ray luminosities, which are not good for testing our predictions. Here we briefly summarize the results on the few sources in MHD03 that satisfy $L_{\mathrm{X}} \lesssim 0.1 L_{\mathrm{X} \text {, crit }}$. We should keep in mind that large uncertainties exist in the normalizations of both correlations in equations (3) and (6) for individual sources.

$N G C 2841 .-M=10^{8.42} M_{\odot}, L_{\mathrm{X}}=10^{38.26} \mathrm{ergs} \mathrm{s}^{-1} \approx 0.03 L_{\mathrm{X}, \text { crit }}$, and $L_{R}=10^{36} \mathrm{ergs} \mathrm{s}^{-1}$. Equation (3) predicts $L_{R}=10^{36.9} \mathrm{ergs} \mathrm{s}^{-1}$, nearly 10 times higher than observations, while equation (6) predicts $L_{R}=10^{35.9}$, which is close to the observed value.

$N G C 3627 .-M=10^{7.26} M_{\odot}, L_{\mathrm{X}}<10^{37.6} \mathrm{ergs} \mathrm{s}^{-1} \approx 0.07 L_{\mathrm{X}, \text { crit }}$, and $L_{R}=10^{36.74} \mathrm{ergs} \mathrm{s}^{-1}{ }^{5}$ Equations (3) and (6) predict $L_{R}<$ $10^{35.55}$ and $L_{R}<10^{34.76} \mathrm{ergs} \mathrm{s}^{-1}$, respectively, both of which are significantly smaller than the observed value.

Sgr $A^{*} .-M=10^{6.41} M_{\odot}, L_{\mathrm{X}}=10^{33.34} \mathrm{ergs} \mathrm{s}^{-1} \approx 10^{-4.8} L_{\mathrm{x}, \text { crit }}$, and $L_{R}=10^{32.5} \mathrm{ergs} \mathrm{s}^{-1}$. Like M31, this should also be a good source to test our prediction, given its extremely low $L_{X}$. The predicted radio luminosity from equation (3) $\left(L_{R} \sim 10^{32.3} \mathrm{ergs} \mathrm{s}^{-1}\right)$ is much closer to the observed value than that from equation (6) $\left(L_{R} \sim 10^{29.3} \mathrm{ergs} \mathrm{s}^{-1}\right)$, which is opposite to our expectation. On the other hand, it is well known that $\mathrm{Sgr} \mathrm{A}^{*}$ is a special radio source (e.g., Falcke \& Markoff 2000). Unlike the typical core-jet AGNs, Sgr A* is observed to be quite compact (e.g., Lo et al. 1998). One possibility is that there is no jet in Sgr $\mathrm{A}^{*}$; thus, our assumption of the existence of jets fails. In this case, the radio emission in $\mathrm{Sgr} \mathrm{A}^{*}$ may come from nonthermal electrons in the $\operatorname{ADAF}$ (Yuan et al. 2003). If a jet does exist in Sgr A*, the powerlaw energy distribution of electrons in the jet $\left[N(\gamma) \propto \gamma^{-p}\right]$ must be unusually steep, e.g., $p>3,{ }^{6}$ as argued by Falcke $\&$ Markoff. Such a steep distribution results in an unusually high radio/X-ray ratio, consistent with the observed low luminosities at infrared and X-ray bands. It may be instructive to compare Sgr A* to

\footnotetext{
${ }^{5}$ We recalculate $L_{R}$, using a new distance consistent with that used in calculating $L_{\mathrm{X}}$.

${ }^{6}$ Both the theoretical studies of shock acceleration and the observed, optically thin, radio synchrotron spectra of extended sources suggest $p \gtrsim 2$. We use $p \approx 2.2$ in the present paper, as in YCN05.
} 
M31. Compared to M31, the mass of the black hole in Sgr A* is 10 times lighter, but $L_{R}$ is even higher, and $L_{\mathrm{X}}$ is more than 100 times lower.

$M 32 .-M=10^{6.4} M_{\odot}$ and $L_{\mathrm{X}}=10^{35.97} \mathrm{ergs} \mathrm{s}^{-1} \approx 10^{-2.1} L_{\mathrm{x}, \text { crit. }}$. This would be another good source to test our predictions, but unfortunately there is only an upper limit on $L_{R}<10^{33.3} \mathrm{ergs} \mathrm{s}^{-1}$. Equation (3) predicts $L_{R}=10^{33.9} \mathrm{ergs} \mathrm{s}^{-1}$, which seems a bit too high, while equation (6) predicts $L_{R}=10^{32.5} \mathrm{ergs} \mathrm{s}^{-1}$, which is in better agreement with the observed value. Future radio measurements may provide more stringent tests.

In summary, the current data from AGNs are so far inconclusive, and more radio and X-ray observations of low-luminosity sources are required.

\subsection{Origin of X-Ray Emission in the "Quiescent State"}

We predict that below $L_{\mathrm{X} \text {,crit }}$, the X-ray spectrum should be dominated by the emission from the jet. This prediction provides a good theoretical frame for understanding an otherwise puzzling observational result for M87. The X-ray emission of M87 is usually modeled by an ADAF (e.g., Fabian \& Rees 1995; Reynolds et al. 1996). However, a subsequent Chandra observation strongly implies that the emission is dominated by the jet, as argued by Wilson \& Yang (2002) based on the similarity of the X-ray spectra between the nucleus and jet knots. The jet dominance in M87 is, in our model, because its X-ray luminosity $L_{\mathrm{X}} \sim 0.8 L_{\mathrm{X} \text {,crit. }}$

Another way to test our prediction is therefore to examine the shape of the X-ray spectrum in the "quiescent state" (defined here as black hole sources with $L_{\mathrm{X}} \lesssim L_{\mathrm{X}}$, crit). In general, the $\mathrm{X}$-ray spectrum of a jet emission has roughly a power-law shape. On the other hand, if the emission is dominated by an ADAF, as proposed by Narayan et al. (1996) for the quiescent state of BHCs, the X-ray spectrum should be curved due to the Compton scattering by thermal electrons when $\dot{M}$ is very low, as shown, e.g., in McClintock et al. (2003) and in Figure $3 a$ of the present paper for the quiescent state of XTE J1118+480. Unfortunately, the X-ray data of black hole sources in the "quiescent state" are not of sufficient quality to discriminate between the models. Thus, this important test awaits deep X-ray observations with state-of-the-art instruments like those on X-Ray Multi-Mirror Mission (XMM-Newton).

Figure $3 a$ shows our prediction on the quiescent state spectra of XTE J1118+480. In the model, the mass-loss rate of the jet is $\dot{M}_{\text {jet }}=6 \times 10^{-8} \dot{M}_{\text {Edd }}$, which is assumed to be $\sim 15 \%$ of the accretion rate in the underlying ADAF. Except for $\dot{M}$ and $\dot{M}_{\text {jet }}$, all other model parameters remain the same as in YCN05. We can see from the figure that the $\mathrm{X}$-ray emission of the quiescent state is dominated by the jet. We predict a power-law X-ray spectrum with photon index of $\sim 2$, which is in good agreement with the current best fit of the observational result (McClintock et al. 2003). We also note that the photon indices of other quiescent BHCs are also 2 (Kong et al. 2002; McClintock et al. 2003). Another issue we would like to mention, as pointed out by McClintock et al. (2003), is the mass accretion rate in the quiescent XTE J1118+480. Assuming that the optical emission comes from a truncated thin disk with an inner radius $R_{\mathrm{tr}}$, the value of $R_{\mathrm{tr}}$, which is $\sim 1500 R_{\mathrm{S}}$, can be determined from the optical flux. Combining this result together with the disk instability theory for the outburst, we can estimate the mass accretion rate of the ADAF, which is $\dot{M} \lesssim 10^{-6} \dot{M}_{\text {Edd }}$. However, an ADAF with such an accretion rate would underpredict the $\mathrm{X}$-ray flux by nearly 4 orders of magnitude (ref. Fig. $3 a$ ). On the other hand, if the X-ray flux is from the jet as we suggest above, there will be no such problem, because this accretion rate is $\sim 20$ times higher than the above $\dot{M}_{\text {jet }}$. Of course, if the optical flux is generated by the impact of the stream from the companion star on the disk surface, there will be no such constraint on $\dot{M}$ (McClintock et al. 2003).

\section{DISCUSSION}

Fender et al. (2003; see also Gallo et al. 2003) compared the power of the jets, $P_{\text {jet }}$ (as inferred indirectly from the radio luminosity $L_{R}$ ), and the X-ray luminosity, $L_{\mathrm{X}}$, of BHCs. Extrapolating $L_{R} \propto L_{\mathrm{X}}^{0.7}$ to low luminosities, they showed that when the $\mathrm{X}$-ray luminosity is below a critical value, $P_{\text {jet }}$ should be greater than $L_{\mathrm{X}}$. The implication of this result is, however, not clear. For instance, it does not mean that the quiescent state X-ray emission of BHCs is dominated by the jets, which is what we conclude in the present work. Moreover, we predict that the radio-X-ray correlation becomes much steeper at low luminosities. In addition, the X-ray luminosity of XTE J1118+480 in outburst is far above their critical luminosity $\left(L_{\text {crit }} \sim 4 \times 10^{-5} L_{\text {Edd }}\right)$, so $P_{\text {jet }}$ should be much smaller than $L_{\mathrm{X}}$ according to their prediction. However, our calculation (YCN05) shows that $P_{\text {jet }} \approx 2 L_{\mathrm{X}}$, and Malzac et al. (2004) obtain $P_{\text {jet }} \approx 10 L_{\mathrm{X}}$.

In the quiescent state, BHCs seem to be much less luminous than their neutron star counterparts (e.g., Garcia et al. 2001; McClintock et al. 2003). Narayan et al. (1997; see also McClintock et al. 2004) take this as evidence for the existence of event horizons in BHCs for the following reasons. For neutron star systems, the energy stored in the ADAF should eventually be released as radiation upon impacting the solid surface of the neutron star. The radiative efficiency is $\sim G M / R_{*} c^{2} \sim 0.15$. For BHCs, however, the energy stored in the ADAF simply disappears into the event horizon of the black hole, so the luminosity is expected to be much lower. Even if the luminosity of BHCs is dominated by the emission from jets, this argument can still hold. Turning the argument around, the systematic difference in the observed X-ray luminosities of black hole and neutron star systems in the quiescent state poses a constraint on our model. In the jet-dominated case, the radiative efficiency of the whole system will be $q_{\text {jet, rad }} \eta$, with $q_{\text {jet, rad }}$ being the radiative efficiency of the jet. To explain the difference of a factor of $\sim 100$ between the luminosities of BHCs and their neutron star counterpart (see Fig. 16 in McClintock et al. 2003), $q_{\text {jet, rad }} \eta$ must be $\sim 0.0015$. Given $q_{\text {jet, rad }} \sim 0.05(\mathrm{YCN} 05)$ and $\eta \sim 10 \%$ or $1 \%$ (see Fig. 2), we have $q_{\text {jet, rad }} \eta \sim 0.005$ or 0.0005 , which are comparable to the required value.

\section{SUMMARY}

The main conclusions from this work can be summarized as follows: (1) Our accretion-jet model developed in YCN05 can reproduce the observed radio-X-ray correlation with an index of 0.7 (Fig. 2). (2) Assuming that the jet persists, we predict that below a critical X-ray luminosity $\left(L_{X, \text { crit }}\right)$ defined in equation (7), the radio-X-ray correlation should turn steeper, from equation (3) to equation (6) (Fig. 1). (3) A related prediction is that the X-ray emission of a source is dominated by that from the jet, when its $\mathrm{X}$-ray luminosity is below $L_{\mathrm{X} \text {, crit }}$. This is particularly relevant to the X-ray emission of BHCs in the quiescent state and some "quiescent" AGNs (whose X-ray luminosity $\lesssim L_{X \text {, crit }}$ ) (Fig. 3).

We thank J. E. McClintock for providing us with the data of XTE J1118+480. This work was supported in part by NASA grant NAG5-9998. 


\section{REFERENCES}

Blandford, R. D., \& Königl, A. 1979, ApJ, 232, 34

Corbel, S., Nowak, M. A., Fender, R. P., Tzioumis, A. K., \& Markoff, S. 2003, A\&A, 400, 1007

Crane, P. C., Dickel, J. R., \& Cowan, J. J. 1992, ApJ, 390, L9

Esin, A. A., McClintock, J. E., \& Narayan, R. 1997, ApJ, 489, 865

Fabian, A. C., \& Rees, M. J. 1995, MNRAS, 277, L55

Falcke, H., Körding, E., \& Markoff, S. 2004, A\&A, 414, 895

Falcke, H., \& Markoff, S. 2000, A\&A, 362, 113

Fender, R. P. 2005, in Compact Stellar X-Ray Sources, ed. W. H. G. Lewin \& M. van der Klis (Cambridge: Cambridge Univ. Press), in press (astro-ph/ 0303339)

Fender, R. P., Gallo, E., \& Jonker, P. G. 2003, MNRAS, 343, L99

Gallo, E., Fender, R. P., \& Pooley, G. G. 2003, MNRAS, 344, 60

Garcia, M. R., McClintock, J. E., Narayan, R., Callanan, P., Barret, D., \& Murray, S. S. 2001, ApJ, 553, L47

Garcia, M. R., Williams, B. F., Yuan, F., Kong, A. K. H., Primini, F. A., Barmby, P., Kaaret, P., \& Murray, S. S. 2005, ApJ, in press (astro-ph/ 0412350)

Heinz, S. 2005, MNRAS, in press (astro-ph/0409029)

Heinz, S., \& Sunyaev, R. A. 2003, MNRAS, 343, L59

Jonker, P. G., Gallo, E., Dhawan, V., Rupen, M., Fender, R. P., \& Dubus, G. 2004, MNRAS, 351, 1359
Kong, A. K. H., McClintock, J. E., Garcia, M. R., Murray, S. S., \& Barret, D. 2002, ApJ, 570, 277

Lo, K. Y., Shen, Z. Q., Zhao, J. H., \& Ho, P. T. P. 1998, ApJ, 508, L61

Malzac, J., Merloni, A., \& Fabian, A. 2004, MNRAS, 351, 253

Markoff, S., Falcke, H., \& Fender, R. 2001, A\&A, 372, L25

Markoff, S., Nowak, M., Corbel, S., Fender, R., \& Falcke, H. 2003, A\&A, 397, 645

McClintock, J. E., Narayan, R., Garcia, M., Orosz, J. A., Remillard, R. A., \& Murray, S. S. 2003, ApJ, 593, 435

McClintock, J. E., Narayan, R., \& Rybicki, G. B. 2004, ApJ, 615, 402

Merloni, A., Heinz, S., \& Di Matteo, T. 2003, MNRAS, 345, 1057 (MHD03)

Narayan, R. 2005, Ap\&SS, in press (astro-ph/0411385)

Narayan, R., Garcia, M. R., \& McClintock, J. E. 1997, ApJ, 478, L79

Narayan, R., McClintock, J. E., \& Yi, I. 1996, ApJ, 457, 821

Reynolds, C. S., Di Matteo, T., Fabian, A. C., Hwang, U., \& Canizares, C. R. 1996, MNRAS, 283, L111

Wilson, A. S., \& Yang, Y. 2002, ApJ, 568, 133

Yuan, F., Cui, W., \& Narayan, R. 2005, ApJ, 620, 905 (YCN05)

Yuan, F., Quataert, E., \& Narayan, R. 2003, ApJ, 598, 301 